\title{
BRANCHED SURFACES AND ATTRACTORS. I: DYNAMIC BRANCHED SURFACES
}

\author{
JOE CHRISTY
}

\begin{abstract}
We show how, using ideas of R. F. Williams about branched surfaces, hyperbolic attractors of flows on three manifolds may be classified up to topological equivalence on an isolating neighborhood by a finite combinatorial object, a swaddled graph.
\end{abstract}

\section{INTRODUCTION}

In 1960, Smale [Sm1, Sm2], inspired by recent work of Peixoto on surfaces [P1, P2], set the following twofold goal for Dynamical Systems: to find a reasonable equivalence relation among flows on a manifold and an open and dense subset of the space of all flows, for which the equivalence classes may be classified by numerical and algebraic invariants. A leading candidate for such an equivalence relation is the notion of topological equivalence. Two flows, $\phi$ and $\psi$, are said to be topologically equivalent, if there is a homeomorphism of the underlying space which takes the oriented, unparametrized orbits of $\phi$ to the oriented, unparametrized orbits of $\psi$. A flow $\phi$ is called structurally stable, if any sufficiently close flow $\psi$ is topologically equivalent to $\phi$. The equivalence class of a structurally stable flow is open; the qualitative dynamical features of a structurally stable flow are insensitive to the inaccuracies of our observation. While many examples are now available to demonstrate the nondensity of structurally stable flows on manifolds of dimension greater than two, e.g. [AS, GW], these flows are the largest class for which a discrete classification up to topological equivalence is possible. Moreover, the theorem of Robbin-Robinson [Rob], gives a sufficient geometrical condition for structural stability, which, after Mañé [M], is also necessary when the underlying manifold is three dimensional. We call such flows hyperbolic; we define them carefully below. The purpose of this paper is to give a complete, finite, combinatorial description of two dimensional attractors of hyperbolic flows on three manifolds. Our goal is to produce a list of names for all such attractors which has an understandably small redundancy and therefore effectively classifies these attractors up to topological equivalence on an isolating neighborhood. These names are simple enough to be manipulated algorithmically, reducing the study

Received by the editors December 27, 1990.

1991 Mathematics Subject Classification. Primary 58F12, 58F13, 58F15, 57N10.

Key words and phrases. Attractor, isolating neighborhood, branched surface, swaddled graph, topological equivalence.

Supported, in part, by NSF grant DMS 8610730 (1). 
of the rich dynamics of these attractors to a mere formal exercise. Combined with the work of DeRezende, Frank, Franks, Fried, Williams, and the author on the structure of hyperbolic flows in dimension three on the one hand, and the Structural Stability theorems of Mañé, Robbin and Robinson on the other, it should soon be possible to classify all structurally stable flows in three dimensions. Our construction also gives a plethora of examples of interesting new foliations and laminations of three manifolds, a subject of much recent interest to geometric topologists. In particular, unlike the examples of Gabai, Hatcher, and Oertel, our laminations have no compact leaves, nor admit any transverse measures, since every noncontractible leaf has contracting holonomy. Our program is to exploit relentlessly an old idea of Williams [Wms], namely to collapse a well chosen isolating neighborhood of a two dimensional attractor along the one dimensional stable foliation to obtain a branched surface. For us, a branched surface, crudely speaking, will be a two dimensional CW complex, with a restricted sort of nonmanifold set, the branch locus, and well defined tangent plane everywhere. It turns out that among all branched surfaces, the 'dynamic' branched surfaces that arise from such a collapsing have a rather simple topological structure, which is imposed upon them by the ghosts of the original flow and stable manifold foliation. The nonsingularity of the flow constrains the tangent bundle, while the one-dimensionality of the stable manifold foliation requires the existence of a normal line bundle. This means that dynamic branched surfaces are determined by a regular neighborhood of their branch loci supplemented sometimes by an identification of the line bundles over certain boundary components of the regular neighborhood. This neighborhood and identification, in turn, are completely determined by the 4-valent graph underlying the branch locus and a labeling on the vertices, edges and certain cycles which reflects the structure of the tangent bundle and a normal line bundle to the branched surface. These objects, which we will call tidy swaddled graphs, are the combinatorial names for attractors that make up our list. The original flow survives as a map from the underlying 4-valent graph to itself and remarkably enough, the dynamics of this map are controlled by the topology of dynamic branched surface and hence captured in the tidy swaddled graph. In this paper we will review the correspondence between certain branched surfaces and attractors, characterize these branched surfaces among all branched surfaces, and discuss their dynamics and relationship to tidy swaddled graphs. We will see that to each tidy swaddled graph on our list there is a unique hyperbolic attractor; in particular, we have the following theorems.

Theorem 3.1. A three dimensional isolating neighborhood $\mathbf{M}$ of a two dimensional hyperbolic attractor $\Lambda$ is determined, up to homeomorphism, by the tidy swaddled graph B.

Theorem 4.2. The flow $\phi$ on a hyperbolic attractor $\Lambda$ is determined, up to topological equivalence on the neighborhood $\mathbf{M}$, by $\mathbf{B}$.

This paper is part of a suite of papers. In [C2] we will show how to construct the list of names, by generating all swaddled graphs and then checking for the proper topology and dynamics. We also discuss the redundancy in the list, that is the equivalence relation among swaddled graphs which give rise to 
branched surfaces obtainable by collapsing topologically equivalent flows. This equivalence relation is generated by a few combinatorial moves on the swaddled graph, and the complexity of a swaddled graph may always be minimized after a finite number of moves. There are a finite number of simplest tidy swaddled graphs associated to a particular hyperbolic attractor $\Lambda$ and they may be explicitly derived from any tidy swaddled graph for $\Lambda$. The contents of this paper are organized as follows. In $\S 0$ we review the relevant definitions and theorems from the theories of hyperbolic flows and three dimensional topology. In $\S 1$ we carefully define branched surfaces and discuss collapsing a nice neighborhood of an attractor to a branched surface. In $\S 2$ we prove a version of the Poincare-Hopf index formula for branched surfaces which allows us to characterize those branched surfaces which admit nonsingular semiflows transverse to their branch loci. In $\S 3$ we delve deeper into the topology of the branched surfaces of the previous section and show how the branch locus organizes the rest of the branched surface and tells us how to reconstruct, via exfoliation, a three manifold from the branched surface. In $\S 4$ we will see how the branch locus constrains the possible symbolic dynamics for a semiflow transverse to the branch locus, and allows us to determine when the branched surface admits a topologically transitive semiflow transverse to its branch locus. We also discuss how the branch locus relates to the boundary periodic orbits of the flow. In $\S 5$ we examine the relationship among topologically transitive semiflows, the nonexistence of transverse measures, and incompressible surfaces. This will provide an easy way of detecting certain sorts of simple dynamics, for example Morse-Smale flows, on the exfoliated three manifold. In $\S 6$ we present some simple examples of hyperbolic attractors in orientable three manifolds. Not surprisingly, these include a flow transverse to the fibration of the figure eight knot, which is well known to be obtainable, via the DA construction from the suspension of the standard hyperbolic toral automorphism, $\left(\begin{array}{ll}2 & 1 \\ 1 & 1\end{array}\right)$. We then discuss some examples of tidy swaddled graphs and branched surfaces which cannot arise from hyperbolic attractors in three manifolds. Finally, for contrast, we present an example of an attractor on a nonorientable manifold.

I would like to dedicate this paper to Avril Thorne, without whose inspiration this work would never have begun. It is an honor to acknowledge many useful conversations on this material with John Franks, David Fried, and Bob Williams, without whose support the research would never have been completed. It is a pleasure to thank the referee for helping me understand the true nature of tidy swaddlings. Finally, I would like to thank the Institute for Advanced Study, Boston University, and the University of California, Santa Cruz, for their hospitality during the many revisions of this paper.

\section{BACKGROUND MATERIAL}

Throughout, $\mathbf{M}^{3}$ will be a compact Riemannian three manifold with boundary and $\phi_{t}$ will be a nonsingular flow of class $C^{2}$, transverse to $\partial \mathbf{M}$. Good general references are the texts of Hempel [He] for three dimensional topology and Shub [Sh] for dynamics. Recall that a point $x$ is said to be $\varepsilon$-pseudoperiodic under $\phi$ if there are points $x_{0}=x, x_{1}, \ldots, x_{n-1}, x_{n}=x$, and times $t_{i}$, such that $d\left(\phi_{t_{i}} x_{i}, x_{i+1}\right)<\varepsilon, \forall i=0, \ldots, n-1$. We call a point which 
is $\varepsilon$-pseudoperiodic for all $\varepsilon>0$, chain recurrent, and denote by $\mathscr{R}$ the set of all such points. We say that $\mathscr{R}$ is hyperbolic if the tangent bundle to $\mathbf{M}$ over $\mathscr{R}$ admits a hyperbolic splitting, $T_{\mathscr{R}} \mathbf{M}=E^{\phi} \oplus E^{u} \oplus E^{s}$, where $E^{\phi}$ is the tangent line field to $\phi$, and for some Riemannian metric on $\mathbf{M}, E^{u}$ and $E^{s}$ are respectively exponentially expanded and contracted by $\phi$, that is, there are $\lambda>1$ and $c>0$ such that for all future times $t>0$ and all vectors $v$ in $E^{u},\left\|\left(\phi_{t}\right)_{*} v\right\|>c \lambda^{t}\|v\|$, and similarly for $E^{s}$. The Stable Manifold Theorem guarantees that $E^{u}, E^{\phi} \oplus E^{u}, E^{s}$, and $E^{\phi} \oplus E^{s}$ are tangent to $\phi$-invariant laminations $\mathscr{W}^{u}, \mathscr{W}^{\phi u}, \mathscr{W}^{s}$, and $\mathscr{W}^{\phi s}$, the strong unstable, unstable, strong stable and stable laminations, (a lamination of $\mathbf{M}$ is a foliation of a closed subset of $\mathbf{M}$ ). We say that a flow satisfies the strong transversality condition if $\mathscr{W}^{u}$ is transverse to $\mathscr{W}^{\phi s}$ everywhere. Finally we say that a flow is hyperbolic if its chain recurrent set is hyperbolic and it satisfies the strong transversality condition. Two flows, $\phi$ and $\psi$, are said to be topologically equivalent, if there is a homeomorphism of the underlying space which takes the orbits of $\phi$ to the orbits of $\psi$. A flow $\phi$ is called structurally stable, if any sufficiently close flow $\psi$ is topologically equivalent to $\phi$. The theorem of Robbin and Robinson asserts that a hyperbolic flow is structurally stable and the theorem of Mañé gives the converse in dimension three. Smale's spectral decomposition theorem says that the chain recurrent set of a hyperbolic flow decomposes uniquely as a disjoint union of compact, $\phi$-invariant basic sets, $\Lambda_{i}$, each containing a dense orbit. Recall that a flow on a space which has a dense orbit is said to be (topologically) transitive. A basic set, $\Lambda$, as above with the additional property that it has a neighborhood $\mathscr{U}$ such that $\bigcap_{t>0} \phi_{t} \mathscr{U}=\Lambda$, will be called a (hyperbolic) attractor. A smooth, closed neighborhood $\mathbf{M}$ as above, with boundary transverse to $\phi$, and $\Lambda$ contained in the interior of $\mathbf{M}$, is said to be isolating. Note that any two isolating neighborhoods of an attractor are isotopic along the flow. It is easy to see that for a hyperbolic flow the attractors are either fixed points, isolated closed orbits, or two dimensional, so-called strange attractors. These last will be our main concern in this paper; it is our hope that they will no longer remain strange.

\section{BRANCHED SURFACES AND COLLAPSING}

Our definition differs slightly from other definitions in the literature; it is more limited than [Wms], since we need only consider the generic case of Williams' definition, and more general than [O], since we do need to consider transversely nonorientable codimension one laminations and nonorientable ambient manifolds.

Definition 1.1. A branched surface, $\mathbf{W}$, is a two dimensional complex which is locally homeomorphic, via orientation preserving charts $\phi$, to a neighborhood on one of the following two models. Furthermore, the transition maps for these charts are required to be smooth.

(right) $\left\{\begin{array}{l|l}(x, y, z) \in \mathbb{R}^{3} & \begin{array}{r}z=e^{-(x-y)^{-2}}, \quad y-x>0 \\ \text { or } z=0 \\ \text { or } z=-e^{-(x+y)^{-2}}, y+x>0\end{array}\end{array}\right\}$, 

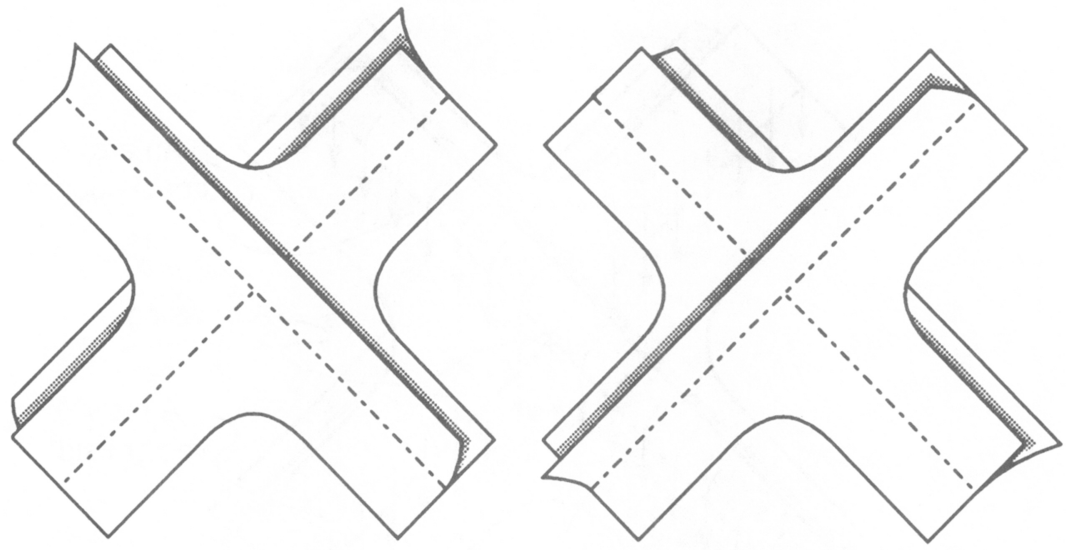

FIGURE 1.1. Left and right crossings
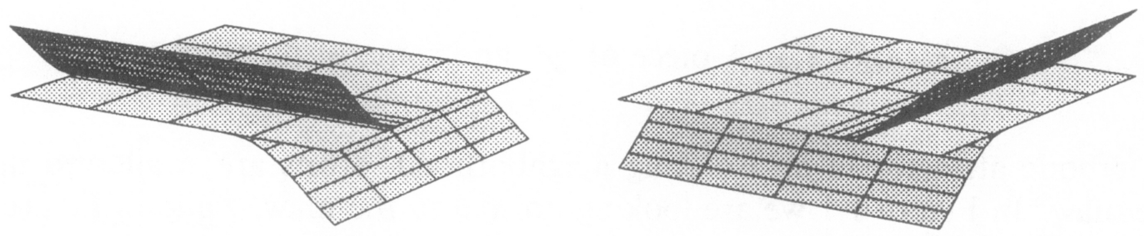

FIGURE 1.2. Left and right crossings, after Williams

or

(left)

$$
\left\{\begin{array}{l|l}
(x, y, z) \in \mathbb{R}^{3} & \begin{array}{l}
z=e^{-(x+y)^{-2}}, \quad y+x>0 \\
\text { or } z=0 \\
\text { or } z=-e^{-(x-y)^{-2}}, y-x>0
\end{array}
\end{array}\right\} .
$$

One should compare Figure 1.1 with the traditional pictures of Williams [Wms] in Figure 1.2, which unfortunately obscure some of the geometry which is crucial to our later arguments.

The branched surfaces of interest to us will have a natural embeddings in three manifolds, and hence we will need to speak of their normal line bundles.

Definition 1.2. A normal line bundle $\nu$ to a branched surface $\mathbf{W}$ is a space locally diffeomorphic to the $\mathbb{R}$ bundle spanned by $\frac{\partial}{\partial z}$ over one of the local models above, with the transition maps for these charts preserving the vertical direction.

We now introduce some terminology, illustrated in Figure 1.3. We call points sent by $\phi$ to $(0,0,0)$, crossings, and the set of points sent by $\phi$ to the rays $x= \pm y, x \neq 0$, arcs of double points. Together they form the branch locus, $\mathbf{B}$, of $\mathbf{W}$. When $\mathbf{W}$ is compact, $\mathbf{B}$ will be a union of smooth circles with normal crossings. We will denote by $\mathscr{Y}$ a small, smooth, closed regular neighborhood of $\mathbf{B}$ in $\mathbf{W}$. We will refer to the components of $\mathbf{W} \backslash \operatorname{int}(\mathscr{Y})$ as the sectors of $\mathbf{W}$. On $\mathscr{Y}$ there is a natural homotopy class of nonsingular tangent vector fields, namely those which are everywhere transverse to $\mathbf{B}$ and point from the locally two-sheeted side to the one-sheeted side of $\mathbf{B}$ in $\mathbf{W}$. We will call any such vector field a maw vector field. In the dynamic branched surface which caricatures a 


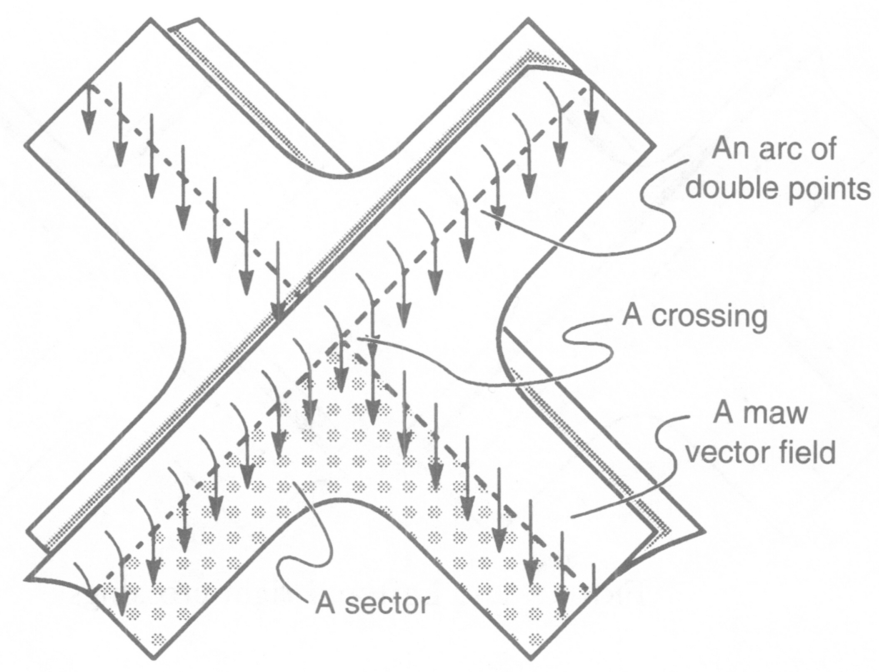

Figure 1.3. A piece of $\mathscr{Y}$ and some of its parts

hyperbolic attractor in an isolating neighborhood, orbits are swallowed up in the maw. In Figure 1.1 we are looking normal to the maw, while in Figure 1.2 we are looking down it.

Away from the crossings, $\mathscr{Y}$ will be a triod- or $Y$-bundle over $\mathbf{B}$, with one of the angles pinched between the top and bottom of the $Y$; in a neighborhood of a crossing, $\mathscr{Y}$ will be homeomorphic to the cone on the 1-skeleton a tetrahedron, with the dihedral angles pinched consistently with the pinching of the $\mathscr{Y}$ bundle structure over the adjacent edges of $\mathbf{B}$. In the tangent bundle to $\mathbf{W}$, the complement to the tangent line to any smooth circle in $\mathbf{B}$ will be oriented by choosing the locally one-sheeted side of $\mathbf{W}$, so the circles of $\mathbf{B}$ will always be orientation preserving curves in $\mathbf{W}$. Note that the normal direction to the pinching of the $Y$-bundle structure of $\mathscr{Y}$ over $\mathbf{B}$ determines, and is consistent with, exactly one line bundle $\nu$ over $\mathscr{Y}$, up to homotopy. An orientation of $\nu$ over a smooth circle $\mathbf{C}$ in $\mathbf{B}$ is the same as a consistent choice of one of the two tangent branches of the $\mathscr{Y}$, so the bundle over $\mathbf{C}$ is nonorientable precisely when there is globally only one sheet at the top of the $Y$; this occurs, for example, near the branch locus of the suspension of the doubling map $z \mapsto z^{2}$ on the circle $\{z \mid\|z\|=1\}$. Figure 1.4 shows an immersion of this $\mathscr{Y}$ in $\mathbf{R}^{3}$. For a more detailed examination of this phenomenon, see [C3].

For a codimension one hyperbolic attractor we construct $\mathbf{W}$ by collapsing $\mathbf{M}$ along $\mathscr{W}^{s}$, and choosing $\nu$ to be the quotient of $E^{s}$. That a branched surface as above arises and not an object with wilder branching, is equivalent to $\partial \mathbf{M}$ and $\mathscr{W}^{s}$ being in general position. Standard techniques [Wms] allow one to arrange this by replacing a given $\mathbf{M}$ with a homeomorphic submanifold. We will assume that this has been done and further, that $\mathbf{M}$ has been isotoped by flowing forward along $\phi$ so long that good hyperbolic estimates hold throughout $\mathbf{M}$. Since $\mathscr{W}^{s}$ is $\phi$-invariant, $\phi$ descends to a nonsingular semiflow $\sigma$ on $\mathbf{W}$. The fact that $\phi$ is transverse to $\partial \mathbf{M}$ and uniformly contracts plaques of $\mathscr{W}^{s}$ implies that the vector field which generates $\sigma$ will be transverse to $\mathbf{B}$ and flow down $\mathscr{Y}$ from the locally two-sheeted side to the locally one-sheeted side, in 


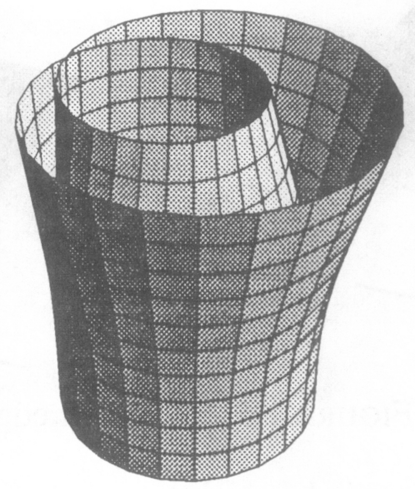

FIgURE 1.4. The Möbius $Y$-bundle

other words, the generator is a maw vector field. Since $\phi$ is hyperbolic, $\sigma$ will be expanding, in other words $\sigma_{*}$ exponentially expands the lengths of vectors in $T \mathbf{W} / \mathbb{R} \frac{d \sigma}{d t}$. Since $\phi$ is topologically transitive, $\sigma$ will also be transitive. For brevity, we will refer to such a triple $(\mathbf{W}, \nu, \sigma)$ of a branched surface, normal line bundle, and an expanding, transitive, nonsingular semiflow transverse to its branch locus, as a dynamic branched surface. Notice that the nonsingularity and expanding character of $\sigma$ imply that no ordinary surfaces are in this class.

In the next section we will see that, for a dynamic branched surface, the topology of the sectors is so simple that $\left.\nu\right|_{y}$ has at most one extension across the sectors, so that there is only one possible $\nu$, given $\mathbf{B}$; moreover, in light of the embedding theorem in [C3], the case where $\mathbf{B}$ consists of a single component without crossings cannot occur. Note that $\mathbf{B}$ will be the disjoint union of a 4-valent graph, that is, a graph with precisely four edges incident on each vertex, and a number of smooth circles. We think of a neighborhood in $\mathbf{B}$ of a crossing lifted to the tangent plane to $\mathbf{W}$ at the crossing, and first we label $\mathbf{B}$ in the quadrant where $\mathbf{W}$ has three sheets with either an $R$ or $L$, corresponding to the handedness of the crossing. Next we choose a maximal tree in $\mathbf{B}$ and consider the remaining edges, which generate $\pi_{1}(\mathbf{W})$. Each of these that reverses the orientation of $\nu$ (which gives the transverse orientation to $\mathbf{W}$ ) while preserving the orientation of $\mathbf{W}$, will be marked with an $M$, for mirror. From this much of the swaddled graph one recovers the topology of $\mathscr{Y}$ by replacing each vertex by the appropriate local model for a crossing, and joining these by $Y$-shaped bands, identifying the ends of the lower branches of the $Y$ 's and preserving or reversing the cyclic order of the upper two branches, according as the corresponding edge of $\mathbf{B}$ has a mirror or not. See Figure 1.5 for the swaddling of edges; note that on the right, $\mathscr{Y}$ is merely immersed, not embedded in $\mathbf{R}^{3}$.

Definition 1.3. A swaddled graph $\mathbf{B}_{s}$ is a graph $\mathbf{B}$, all of whose vertices are 4valent, which may have components which are circles without vertices, together with the following additional data:

(1) an embedding of a neighborhood of each vertex into a neighborhood of the origin in the plane, and an $R$ or an $L$ in one of the quadrants that this embedding determines,

(2) $M$ 's on some of the edges outside of a maximal tree. 

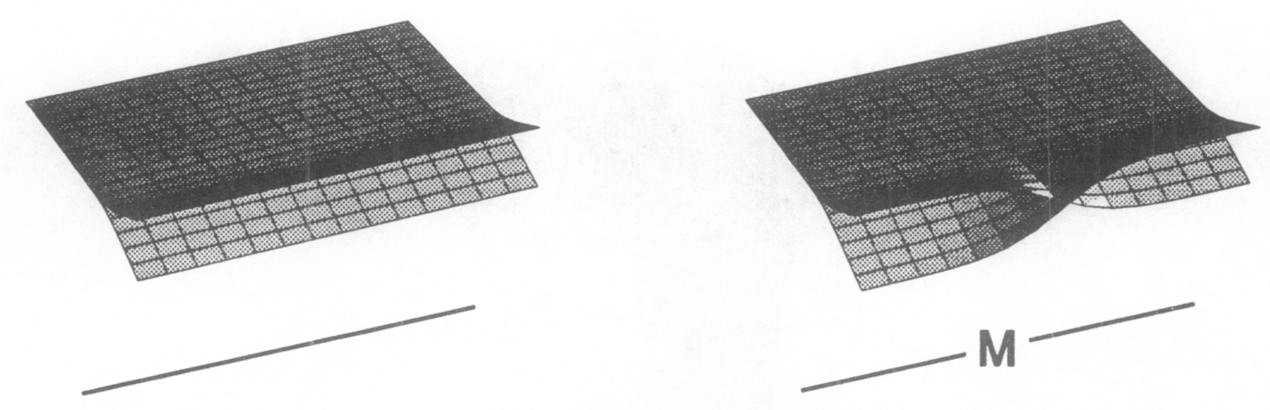

FIGURE 1.5. Swaddled edges

A swaddled graph is bundled as tightly as a newborn infant; the underlying graph is the base of two line bundles.

It is clear that the swaddled graph determines $\left.\nu\right|_{y}$. Notice that $\mathscr{Y}$ can have boundary components which lie entirely on the locally two-sheeted side of $\mathbf{B}_{s}$ or entirely on the locally one-sheeted side of $\mathbf{B}_{s}$. We call these boundary components dangling incoming and outgoing boundary circles, respectively.

Definition 1.4. A tidy swaddled graph is one with a one-to-one pairing of the cycles of $\mathbf{B}_{s}$ homotopic in $\mathscr{Y}$ to boundary components which lie entirely on the locally two-sheeted side of $\mathbf{B}_{s}$ with those homotopic to boundary components which lie entirely on the locally one-sheeted side of $\mathbf{B}_{s}$, each pair labeled by an ordered pair $(t, n)$, where $t$ is an element of $\{+,-\}$ and $n$ is an element of $\{+, 0,-\}$.

The pairing above tidies up the loose ends at the dangling boundary circles; it is precisely the data needed to fix an extension of $\nu$ over the annular sectors of $\mathbf{W}$. The tidying label specifies a homotopy class of bundle isomorphisms between the restrictions of the normal bundle $\nu$ to the dangling boundary circles; $t$ describes the effect of the isomorphism on the orientation of the base, $\mathbf{W}$, and $n$ describes the effect on the orientation, if any, of the fibers of $\nu$ around the cycle. The geometry of the labeling is discussed in detail in $\S 3$ below.

If $\mathbf{B}_{s}$ has no mirrors, then the only loops that reverse the transverse orientation of $\mathscr{Y}$ are those (necessarily with corners at some of the crossings) around which $\mathscr{Y}$ has an odd number of half twists, in which case the loop reverses the orientation of $\mathscr{Y}$ as well, so that the total space of $\left.\nu\right|_{y}$ is orientable. See Figure 6.3 for an example. Conversely, if $\mathbf{B}_{s}$ has an edge with a mirror, then the corresponding element of $\pi_{1}$ reverses the orientation of $\nu$ and preserves the orientation of $\mathscr{Y}$, so the total space of $\left.\nu\right|_{y}$ is nonorientable. When $\mathbf{B}_{s}$ has no mirrors we will speak of $\mathbf{W}$, by extension, as having no mirrors.

\section{The Poincaré-Hopf INDEX THEOREM FOR BRANCHED SURFACES}

For dynamic branched surfaces, not only does the swaddled graph B encode all the topology of $\mathscr{Y}$, but a version of the Poincaré-Hopf index formula also dictates that the sectors of $\mathbf{W}$ have a particularly simple form. The underlying geometrical truth is the following:

Wringer Lemma 2.1. On any dynamic branched surface, we can always find a small, smooth, closed, regular neighborhood $\mathscr{Y}$ of $\mathbf{B}$ such that the pair $(\mathscr{Y}, \sigma)$ is locally diffeomorphic to the model in Figure 2.1. 


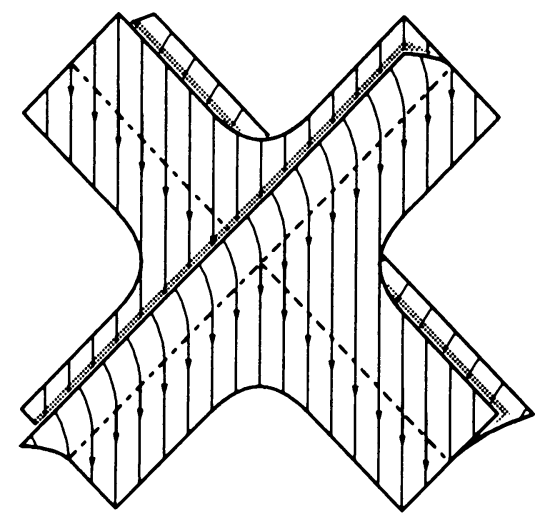

FIGURE 2.1. The Wringer Lemma asserts that the dynamics of $\sigma$ near $\mathbf{B}$ are like laundry passing through a wringer

In particular, $\sigma$ will have precisely two internal tangencies with $\partial \mathscr{Y}$, to the sides of each crossing of $\mathbf{B}$. Conversely, any branched surface has a neighborhood $\mathscr{Y}$ of its branch locus which admits a semiflow $\sigma$ having precisely two internal tangencies with $\partial \mathscr{Y}$ near each crossing of $\mathbf{B}$.

Proof. Work in local coordinates. Notice that the angle between $\sigma$ and B is uniformly bounded away from 0 , so there is no problem along the arcs of double points. Near the crossings, we can use flow box coordinates in which B looks like a pair of transverse smooth arcs and take the boundary of $\mathscr{Y}$ to be given by the branches of two hyperbolae asymptotic to the tangent directions of $\mathbf{B}$ at the crossing.

From this we can deduce the following. Here we abuse our terminology slightly and speak of the sectors of $\mathbf{W}$ as though they were the closures of the components of $\mathbf{W} \backslash \mathscr{Y}$. We also ignore the well-known case where $\mathbf{W}$ is an ordinary surface.

Theorem 2.2. A branched surface $\mathbf{W}$ supports a nonsingular semiflow $\sigma$, transverse to $\mathbf{B}$, if and only if $\chi(\mathbf{W})=0$ (as a two complex), and the sectors of $\mathbf{W}$ are either discs with boundaries doubly externally tangent to $\sigma$, or interval bundles over the circle with boundaries transverse to $\sigma$.

In the first case, we glue the sectors to what we will call bitangent boundary circles of $\mathscr{Y}$, and in the second, to pairs of dangling incoming and outgoing boundary circles. See Figure 2.2 .

$\mathscr{Y}$ in a branched surface which supports a nonsingular $\sigma \pitchfork \mathbf{B}$.

Proof. First, notice that $\chi(\mathbf{W})=\chi(\mathbf{B})+\chi($ sectors), and since $\mathbf{B}$ is 4-valent, $\#$ arcs $=2$ (\#crossings) , so $\chi(\mathbf{B})=$ \#crossings $-\#$ arcs $=-($ \#crossings $)$. Now, the Wringer Lemma shows that the only singularities of $\left.\sigma\right|_{\text {sectors }}$ are two external tangencies with the boundary near each vertex of $\mathbf{B}$. External tangencies with the boundary of a surface have index $\frac{1}{2}$, so applying the ordinary Poincare-Hopf index theorem we can only have surfaces with nonnegative Euler characteristic as sectors, that is discs, annuli and Möbius bands. Moreover, their boundaries must either be transverse to the semiflow in the case of zero Euler characteristic, 


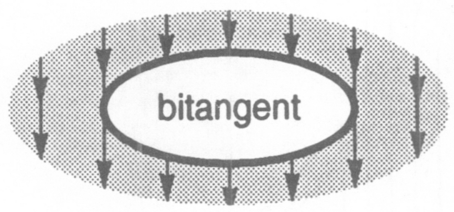

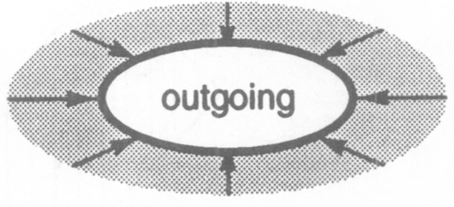

dangling

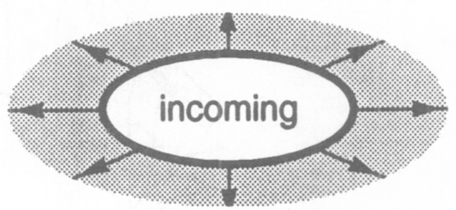

FIGURE 2.2. The possibilities for boundary components of $\mathscr{Y}$ in a branched surface which supports a nonsingular $\sigma \pitchfork \mathbf{B}$

or have precisely two external tangencies in the case of discs; thus we have the $\Rightarrow$ direction. For the $\Leftarrow$ direction, we construct the pair $(\mathbf{W}, \sigma)$ in the obvious way by gluing sectors with flows as above to the neighborhood $\mathscr{Y}$ from the Wringer Lemma.

Lemma 2.3. A dynamic branched surface never has Möbius band sectors.

Proof. Applying the Poincaré-Bendixson theorem to the lift of $\sigma$ to an orientable double cover of such a sector, we see that it must contain an attracting closed orbit, so that the underlying semiflow on $\mathbf{W}$ would not be topologically transitive.

\section{THE TIDY SWADDLED GRAPH DETERMINES THE TOPOLOGY OF A NEIGHBORHOOD OF THE ATTRACTOR}

Next we elucidate the pairing of the outgoing dangling circles with the incoming ones. The pair of labels $(t, n)$ tells us how to join the these circles by an annular sector and extend $\nu$ across the sector. Giving the annulus a product structure $\mathbf{S}^{1} \times \mathbf{D}^{1}$ and thinking of the interval factor as the parameter, an interval bundle over the annulus realizes a homotopy between its restrictions to the boundary components of the annulus. Thus an extension of $\nu$ across an annulus is the same as a homotopy class of isomorphisms of interval bundles over the circle, [Hi]. The description of such homotopy classes is entirely a matter of keeping careful track of orientations, which we proceed to do.

First of all, we orient the boundary circles of $\mathscr{Y}$ by declaring them to be positively oriented when $\mathscr{Y}$ lies on our right as we face forward; put another way, at each point $c$ of a boundary circle $\mathbf{C}$, the ordered pair (inward normal in $\mathscr{Y}$ to $\mathbf{C}$ at $c$, positive tangent to $\mathbf{C}$ at $c$ ) form a positively oriented basis of $T_{c} \mathscr{Y}=\mathbf{R}^{2}$. This construction is unambiguous since the boundary of a surface always has an orientable collar. Having chosen orientations, we can distinguish between the homotopy classes of homeomorphisms of the base circles by their effects on the orientations. With our orientation, a maw vector field will point to the right on dangling incoming boundary circles and to the left 
on dangling outgoing boundary circles. Since $\sigma$ is generated by a maw vector field and extends it across the annular sector in question, the natural maps between paired dangling boundary circles will reverse the orientations of the normals to the circles within their collars in $\mathscr{Y}$, so it makes sense to label the homeomorphisms which reverse the orientations of the circles with $t=+$, since they preserve the orientations of the collars, and to label the other homotopy class by $t=-$. Next, observe that there are two isomorphism classes of interval bundles over the circle. If $\nu$ is nonorientable over both dangling circles, we assign the label $n=0$. If $\nu$ is orientable, then, having specified the effect of the bundle isomorphism on the base orientations, we will use the effect on fiber orientations to distinguish the homotopy classes. We can make this explicit by agreeing that, as we face forward on a boundary circle with orientable $\nu$, the fibers of $\nu$ should be oriented to our left. With this convention, we assign the label $n=+$ when the fiber orientation is preserved, and $n=-$ when it is reversed.

If $\mathbf{W}$ has mirrors, it may not not be possible to tidy the swaddling. For example, in the suspension of the doubling map of the circle (see Figure 1.4) $\mathbf{B}$ is a circle with a single mirror, and there is one sector, which is an annulus. Since the outgoing dangling circle of $\mathscr{Y}$ is homotopic to $\mathbf{B}, \nu$ is a Möbius band there, while over the incoming dangling circle, being homotopic to $2 \mathbf{B}, \nu$ is an annulus. Unfortunately, there is no interval bundle over the annular sector with such behavior over the boundaries. In general, a branched surface embeds in a three-manifold precisely when the total number of mirrors in the branch locus crossed by the boundary of each sector is even [C3]. In $\S 6$ we will give an example of a branched surface which does have mirrors and comes from an attractor in a nonorientable three-manifold.

From a tidy swaddled graph $\mathbf{B}_{s}$ we can now decide whether or not there is a dynamic branched surface $\mathbf{W}$ with branch locus $\mathbf{B}$ and, if there is, uniquely reconstruct $\mathbf{W}$ from $\mathbf{B}_{s}$. This is because the swaddled graph $\mathbf{B}_{s}$ determines $\mathscr{Y}$ and if $\mathscr{Y}$ has boundary circles with four or more essential tangencies, or an imbalance between the number of outgoing and incoming boundary circles, then we have seen that it embeds in no branched surface supporting a nonsingular semiflow, transverse to $\mathbf{B}$. On the other hand, if $\mathbf{B}_{s}$ gives rise to a $\mathscr{Y}$ with allowable tangencies and equal numbers of incoming and outgoing dangling circles, then for any boundary circle of $\mathscr{Y}$, there is exactly one topological type of sector possible, depending on the number of essential tangencies. Moreover, there is exactly one extension of $\nu$ across the sectors, which is dictated by the tidying in the case of annular sectors. Given a dynamic branched surface we can recover a two dimensional hyperbolic attractor $\Lambda$ by exfoliating. This can be thought of as taking the inverse limit of $\mathbf{W}$ under $\sigma$, but it is geometrically equivalent to the following construction, illustrated in Figure 3.1, which also gives an embedding of $\Lambda$ in a three-manifold. Replace the closures of the sectors of $\mathbf{W}$ by the interval bundles over them specified by the swaddling on their boundaries and $\sigma$ by $\phi=(\sigma \times$ a contracting flow on $\mathbf{I}$, transverse inward along $\partial \mathbf{I})$, and then glue over $\mathbf{B}$, consistent with $\left.\nu\right|_{y}$, making $\phi$ smooth and smoothing the boundary in the maw of $\mathbf{W}$. This smoothing will give rise to a circle of tangencies of $\partial \mathbf{M}$ and $\mathscr{W}^{\phi s}$, which we will also refer to as the maw of $\partial \mathbf{M}$.

This produces a three-manifold $\mathbf{M}$ with a flow $\phi$ transverse to $\partial \mathbf{M}$ and 


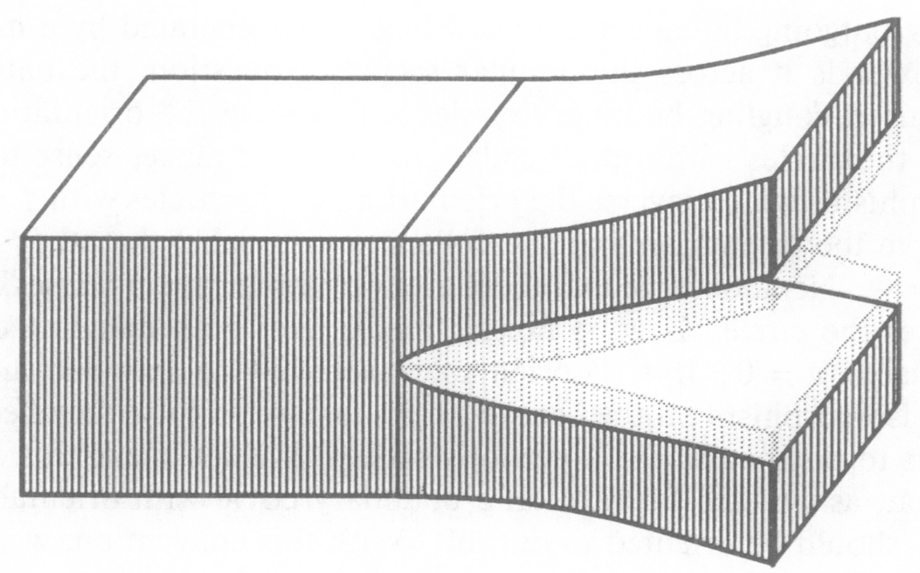

FIGURE 3.1. Thickening near B

chain recurrent set a single attractor. Furthermore, the $\mathscr{W}^{\phi u}$ lamination extends to a foliation $\mathscr{F}$ of $\mathbf{M}$, gotten by saturating the sets $\{$ (interval in $\mathbf{B}) \times$ (point in I) $\}$ by $\phi$. This foliation has no closed leaves and is transverse to $\partial \mathbf{M}$. Since $\mathbf{W}$ is a deformation retract, along $\mathscr{W}^{s}$, of the exfoliated $\mathbf{M}^{3}, \pi_{1}(\mathbf{M})=$ $\pi_{1}(\mathbf{W})$. Now $\pi_{1}(\mathbf{W})$ is presented by the edges of $\mathbf{B}-($ a maximal tree), subject to the relations gotten by killing the words corresponding to doubly tangent boundary circles of $\mathscr{Y}$ and making HNN extensions conjugating the paired dangling circles (here we adjoin a new generator and a new relation saying that the new generator conjugates the two words in the old generators corresponding to the dangling circles [He]). Since $\mathbf{M}^{3}$ has a boundary, a standard argument constructs incompressible surfaces [He]; since it supports a foliation transverse to its boundary without closed leaves, a theorems of Novikov [N] and RoussarieThurston [Rou] tells us that it is irreducible. Therefore $\mathbf{M}$ is Haken. We can present $\pi_{1}(\partial \mathbf{M})$ as a subgroup of $\pi_{1}(\mathbf{M})$ as above, only now the relations come from the $\partial \mathbf{I}$ bundles over the sectors, rather than the sectors themselves. Therefore, by Waldhausen's theorem [Wald, He], $\mathbf{M}$ is determined up to homeomorphism by its fundamental group and peripheral subgroups, which, we have just seen are determined by $\mathbf{B}_{s}$, hence

Theorem 3.1. An oriented three dimensional neighborhood of a two dimensional hyperbolic attractor is determined, up to homeomorphism, by the tidy swaddled graph $\mathbf{B}_{s}$.

\section{SWADDLED GRAPHS AND DYNAMICS}

The tangency structure on the boundary circles of $\mathscr{Y}$ (which is determined by $\mathbf{B}$ ) also tells us about the first return map $\rho$ to $\mathbf{B}$, and so allows us to recover the dynamics of $\phi$. This is because the edges of $\mathbf{B}$ can often be refined to give a Markov partition for $\sigma$ whose allowed transitions are determined by the topology of $\mathbf{W}$ and its sectors. At first glance it seems that there may be many dynamically inequivalent Markov partitions consistent with B, but we will see that this is not the case. We will show in Proposition 4.1 below that any two expanding semiflows $\sigma$ and $\sigma^{\prime}$ on $\mathbf{W}$ can be connected via an arc of 
expanding semiflows, so that the exfoliated flows $\phi$ and $\phi^{\prime}$ will be connected by an arc of hyperbolic flows, hence, by structural stability, $\phi$ and $\phi^{\prime}$ will be topologically equivalent.

In light of the simplicity of the proof and the importance of the result, several remarks about this proposition and its analogues in simpler situations are in order. First, while there is no corresponding proposition for general expanding maps, in the one dimensional case, it follows from the convexity of the space of invariant measures and the techniques of Parry [Pa]. Parry's theorem implies that an expanding maps of one manifolds are conjugate to canonical piecewise linear models, and hence, in certain situations, conjugate to one another [Ta]. Notice, however, that $\sigma$ and $\sigma^{\prime}$, will not, in general, be topologically equivalent, since the periodic or aperiodic character of the orbits of the crossings of B in $\mathbf{W}$ can change under arbitrarily small perturbations. Second, the most straightforward analogue of our proof would be for expanding flows or vector fields on ordinary manifolds, but, as we remarked above, there are none.

Proposition 4.1. Any two expanding, nonsingular semiflows on a branched surface may be joined by an arc of expanding semiflows.

Proof. First we must make more precise our notion of an expanding semiflow. We will say that a semiflow on $\mathbf{W}$ is expanding iff there is a Riemannian metric such that for some complement $E$ in $T \mathbf{W}$ of the subbundle generated by $\frac{d \sigma}{d t}$ there are constants $C>0$ and $\lambda>1$ such that $\left\|\left(\sigma_{t}\right)_{*} v\right\|>C \lambda^{t}\|v\|, \forall t>0$, $\forall v \in E$. If this holds for some complement $E$, then clearly it will hold for any other complement $E^{\prime}$, with perhaps a different constant $C^{\prime}$. The property of being expanding is a local one in that, if we can establish that there is some $t_{0}$ such that for every point $w$ of $\mathbf{W}$, the expanding property holds for $w$ for all times less than $t_{0}$, then by compactness and the semigroup property, the expanding property holds for all $w$ and $t$. Suppose that $\sigma$ and $\sigma^{\prime}$ are two expanding semiflows on $\mathbf{W}$. Since $\sigma$ and $\sigma^{\prime}$ are transverse to the branch locus the instantaneous passage across the branch locus has no effect on their expanding character.

We are left then the problem of interpolating between $\sigma$ and $\sigma^{\prime}$ on the sectors, $\mathbf{W} \backslash \mathbf{B}$. The following elegant argument was suggested to us by David Fried. The semiflows on the sectors are unique up to topological equivalence; either we have a flow on a disc which flows from an incoming arc on the boundary to an outgoing arc, and the arcs are separated by a pair of internal tangencies, or a flow on an annulus which flows from one boundary component to the other. On any particular sector then, we may suppose, after conjugating $\sigma^{\prime}$ by diffeomorphisms (necessarily isotopic to the identity since annuli and discs have trivial groups of mapping classes fixing setwise the boundary components), that the orbits of $\sigma$ and $\sigma^{\prime}$ are identical, and the difference between the flows will be reflected in the difference between the induced metrics which exhibit their expanding nature. Therefore, since the preliminary conjugacy is connected to the identity by an arc of diffeomorphisms, showing that $\sigma$ and $\sigma^{\prime}$, when restricted to some sector, may be connected by an arc of expanding flows, is equivalent to showing that if we have a fixed flow $\sigma_{0}$ with fixed complement $E_{0}$ which is expanding relative to two different metrics, then we may connect the metrics by an arc of metrics, relative to all of which, $\sigma_{0}$ is expanding. This is easy though, since we can just linearly interpolate between the given metrics and use 
the smaller of the constants $C$ and $C^{\prime}$ and $\lambda$ and $\lambda^{\prime}$ for the defining estimates.

Finally, in order to ensure that the interpolating arc of expanding flows extend smoothly across the branch locus, we point out that the Wringer Lemma gives topologically equivalent models for both $\sigma$ and $\sigma^{\prime}$ on $\mathscr{Y}$. Just as on the sectors, we interpolate between the metrics which exhibit the expansion of $\sigma$ and $\sigma^{\prime}$ on $\mathscr{Y}$ to produce an arc of topologically equivalent, expanding semiflows on $\mathscr{Y}$ interpolating $\sigma$ and $\sigma^{\prime}$. Using a partition of unity to patch this arc together with the arcs of expanding flows above on the sectors, we are done.

It is worth remarking that when $\mathbf{B}$ has dangling circles, rotating the vector field which generates $\sigma$ as it crosses an annular sector to produce a new semiflow $\sigma^{\prime}$ has the same effect on the global semiflow as fixing the vector field, cutting the sector open along its core and then reglueing with a (possibly fractional) Dehn twist, or earthquake [T]. Since the latter clearly has no effect on the expanding nature of $\sigma$, (measuring the expansion relative to the earthquaked metric shows that we can take $C$ and $\lambda$ constant throughout the twist), the exfoliated flows $\phi$ and $\phi^{\prime}$ will be topologically equivalent. The situation is illustrated in Figure 4.1. In particular, if the rotation is enough to induce a full twist, then the homeomorphism giving the equivalence no longer need be chosen to be isotopic to the identity.

In any case, we may choose a particular expanding semiflow for which the crossings are periodic, and hence the edges of $\mathbf{B}$ can be refined to give a Markov partition. Now the elements of the partition corresponding to the incoming boundary of some sector must map to the elements coming from the outgoing boundary of that sector, preserving their order, and this structure is dictated by B. Since the Markov partition for $\sigma$ will exfoliate to a Markov partition for the flow, and this Markov partition completely describes the dynamics of $\phi$, we have:

Theorem 4.2. The flow $\phi$ on a hyperbolic attractor is effectively determined, up to topological equivalence, by $\mathbf{B}$.

The structure of $\mathbf{W}$ as encoded in $\mathbf{B}$ also tells us about the boundary periodic orbits of $\Lambda$ [C1]. These are the orbits of points that have a component of $\mathscr{W}^{s}(x) \backslash\{x\}$ disjoint from $\Lambda$. It is a theorem that to every component of $\partial \mathbf{M}$ we can associate such points, and that they are necessarily periodic. They are
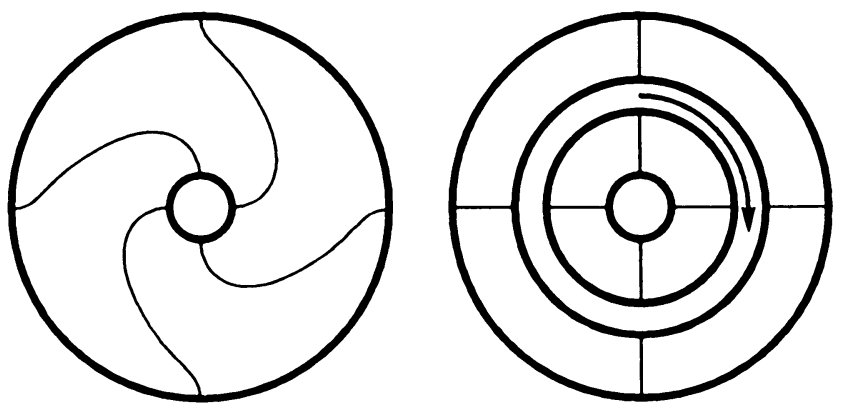

FigURE 4.1. An earthquake about the core of an annular sector is equivalent to rotating the vector field that generates $\sigma$ near the core 

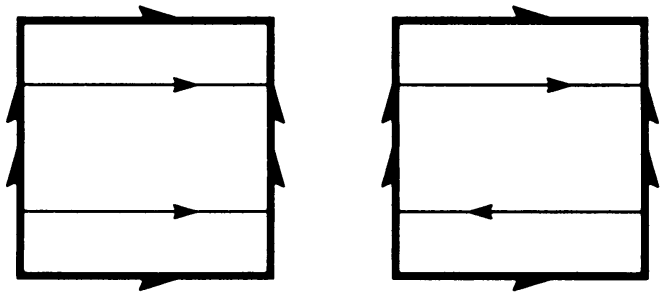

Figure 4.2. A coherent torus, on the left, and an incoherent torus, on the right

a fundamental ambient invariant of a codimension 1 hyperbolic attractor, and in particular they control how $\Lambda$ may be situated relative to other basic sets in a hyperbolic flow, and whether $\Lambda$ has Birkhoff sections (see [C1] and below). From the Poincaré-Hopf index formula, it follows that every component of $\partial \mathbf{M}$ has $\chi=0$, in other words, be either a torus or a Klein bottle. Note that a boundary periodic orbit $\gamma$ homotopes out along its stable manifold to give a homotopically nontrivial, oriented circle $c$ in $\partial \mathbf{M}$. Pushing orientations along $\mathscr{W}^{s}$, we see that when the corresponding component of $\mathbf{M}$ is orientable, $\mathscr{W}^{u}(\gamma)$ is orientable.

Definition 4.3. We say that two boundary periodic orbits for which the corresponding circles are antiparallel, i.e. bound an annulus in $\partial \mathbf{M}$, are incoherent and that the corresponding boundary component is incoherent. If they are parallel, (bound an annulus in $\partial \mathbf{M}$ with the orientation of one reversed) we say that they are coherent. See Figure 4.2.

Since it was shown in [C1] that every component of $\partial \mathbf{M}$ is associated with at least one boundary periodic orbit, all components of $\partial \mathbf{M}$ which are tori or have more than one boundary periodic orbit are either coherent or incoherent.

To discuss the case of $\mathbf{K}^{2}$ boundary components with exactly one boundary closed orbit we must first recall that up to the action of the mapping class group there are three isotopy classes of essential simple closed curves. See Figure 4.3 for a picture; for a proof see the appendix. If we represent $\mathbf{K}^{2}$ as the quotient of $\mathbf{E}^{2}$ by the group generated by a translation and an independent glide reflection, these three classes are represented by the two axes and the boundary of a tubular neighborhood of the axis of the glide reflection.

In the orientation double cover, the axis of the glide reflection lifts to a single curve and the boundary of its neighborhood lifts to two parallel curves while the axis of the translation lifts to two antiparallel curves.

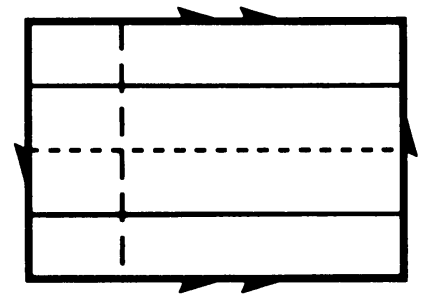

FIGURE 4.3. The three isotopy classes of essential closed curves on the Klein bottle 
Definition 4.3 bis. When the boundary periodic orbits are in the first two classes, we say that $\mathbf{K}^{2}$ is coherent, otherwise it is incoherent. If the components of $\partial \mathbf{M}$ are all coherent, then we refer to $\Lambda$ itself as coherent, otherwise it is incoherent.

Previously, building on earlier work of Bowen and Fried, we had shown that, except for incoherent attractors and repellors, the study of any hyperbolic basic set of a nonsingular Axiom A flow satisfying the strong transversality transversality condition can be reduced to the study of pseudo-Anosov maps of surfaces via the introduction of Birkhoff sections. A Birkhoff section is a slight extension of the ordinary notion of cross section where we allow there to be boundary components parallel to the flow. See [C1] and the references therein. Unfortunately the question of the actual existence of any exceptions was left open; below we will give the first examples of incoherent attractors. The boundary periodic orbits may be difficult to identify directly from the semiflow $\sigma$ on $\mathbf{W}$, however they are intimately related to the maw:

Proposition 4.4. The boundary periodic orbits of $\Lambda$ are homotopic to circles which interleave with the maw, and hence determined by $\mathbf{B}$.

Proof. Consider $\mathscr{W}^{\phi s}(\Lambda) \cap \partial \mathbf{M}$. The maw consists of circles which divide $\partial \mathbf{M}$ into open regions where $\mathscr{W}^{s}$ is transverse to $\partial \mathbf{M}$ and consequently $\mathscr{F}=$ $\mathscr{W}^{\phi s}(\Lambda) \cap \partial \mathbf{M}$ inherits an orientation from $\phi$. Applying Poincaré-Bendixson to the orientation covers of these regions we see that the foliation $\mathscr{F}$ has a closed leaf in each such region. Now the closed leaves of $\mathscr{F}$ cannot bound discs in their leaves of $\mathscr{W}^{\phi s}(\Lambda)$ since these circles, lying in $\partial \mathbf{M}$, are transverse to $\phi$, so that any disc they would bound would have to contain a singularity of $\phi$, a contradiction to our assumption that $\phi$ is nonsingular. Moreover, the circles cannot bound discs in $\partial \mathbf{M}$, since the leaves of $\mathscr{W}^{\phi s}$ are incompressible. Any leaf $L$ of $\mathscr{W}^{\phi s}$ containing a circle $c$ of $\mathscr{F}$ must therefore contain a closed orbit $\gamma$ homotopic to $c$. Now $L$ intersects $\Lambda$ in a closed invariant set and since every orbit of $\phi$ which intersects $c$ has $\gamma$ as its $\omega$-limit set, $\Lambda$ must lie entirely on the other side of $\gamma$ in $L$. In other words $\gamma$ is a boundary periodic orbit.

\section{THE NONEXISTENCE OF INVARIANT TRANSVERSE MEASURES AND INCOMPRESSIBLE SURFACES}

There is another check of the transitivity of $\sigma$ available to us, namely the existence of a nontrivial transverse invariant measure on $\mathbf{W}$. The existence of a transverse invariant measure is expressible in terms of the existence of nontrivial solutions to the branch equations; these express the invariance condition that the weight on the sector adjacent to an edge of $\mathbf{B}$ along the outgoing side must be equal to the sum of the weights on the sectors adjacent to the edge on the incoming side [FO]. See Figure 5.1.

A special case of an invariant measure is the push forward of a Dirac measure concentrated on a surface in $\nu$ transverse to the fibers; these correspond to integral solutions of the branch equations. From our reconstruction of $\mathbf{M}$, we can also think of this surface as embedded in $\mathbf{M}$, where a theorem Oertel [O] tells us that it would be incompressible. This happens, for example, when our flow has a closed invariant surface. 


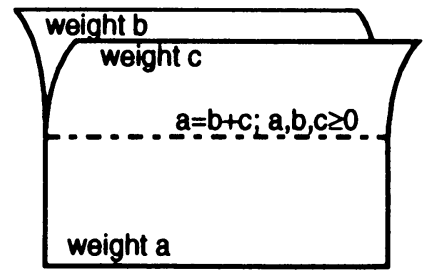

FIGURE 5.1. The branch equations

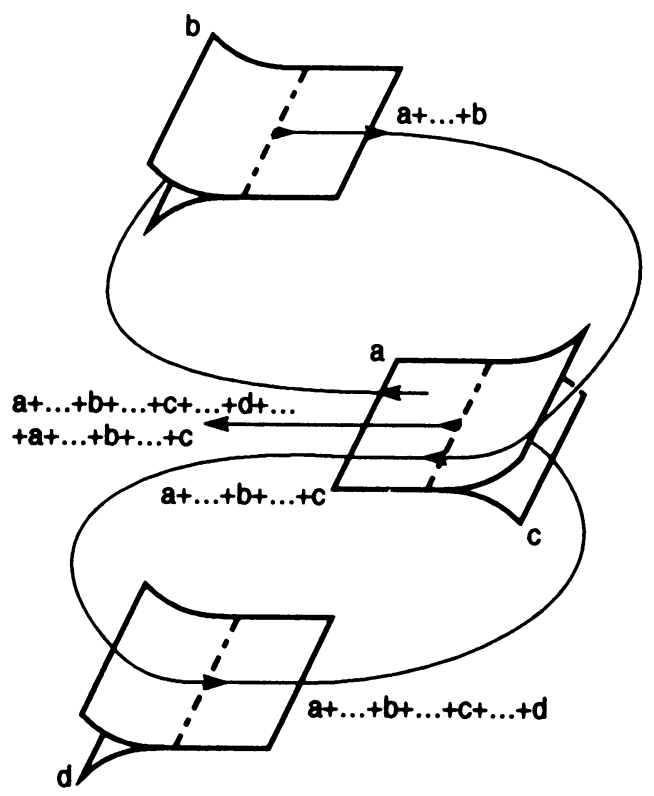

FIGURE 5.2. By successively solving the branch equations as we traverse certain orbits, we see that there are no nontrivial solutions

Proposition 5.1. A dynamic branched surface can admit no nontrivial transverse invariant measures.

Proof. The proof is depicted in Figure 5.2. As we trace the sectors traversed by an orbit of $\sigma$, the branch equations show that each successive sector must have weight greater than the preceding sector, by an amount equal to the weight of the other sector incident on the edge crossed, or the sum of the weights of the other two sectors if the orbit passes through a crossing (these sectors need not be distinct). Consequently, when an orbit first returns to a particular sector, there is an equation of the form

(weight of the sector) + (something nonnegative $)=$ (weight of the sector $)$;

and when it next returns to this sector again, from a different sector than it did in its intermediate return, there will be an equation of the form

2 (weight of the sector) + (something else nonnegative $)=$ (weight of the sector) so the weight of the sector in question must be 0 . The transitivity of the flow, though, allows us to find such an orbit for any sector by first choosing three 
points, the first very near an incoming edge of that sector and the other two close by on the two sectors flowing forward across the edge, then finding an orbit which starts close to the first and then eventually passes close to the other two. Thus there is no transverse invariant measure.

This does not detect all intransitive semiflows, but it is an easy criterion to check, and very efficient in practice.

\section{EXAMPLES}

The best known of all hyperbolic attractors is the suspension of the DA of the Thom automorphism of $\mathbf{T}^{2}$,

$$
\left(\begin{array}{ll}
2 & 1 \\
1 & 1
\end{array}\right)
$$

This flow lives in the complement in $\mathbf{S}^{3}$ of the figure eight knot, and is transverse to the well-known fibration. Its swaddled graph, and a neighborhood of the branch locus are shown in Figure 6.1; Figure 6.2 illustrates the embedding in $\mathbf{S}^{3}$.

There are two doubly tangent boundary circles, and with the labeling above of the edges of $\mathbf{B}$ (the edge 1 is our maximal tree), we obtain the presentation

$$
\pi_{1}\left(\mathbf{M}^{3}\right)=\langle x, y, z \mid x \bar{y} z y \bar{z}=1=x \bar{z} \bar{x} y\rangle,
$$

which can be reduced to

$$
\pi_{1}\left(\mathbf{M}^{3}\right)=\langle y, z \mid z \bar{y} \bar{z} y \bar{z} \bar{y} z y \bar{z} y\rangle,
$$

the two bridge presentation of the knot group. There are two curves in the maw, so there are two boundary periodic orbits, and they are meridians of the knot.

For the return map to $\mathbf{B}$, we have

$$
\rho: x 1 \mapsto y 1 x \bar{z}, \quad \rho: \bar{y} z \mapsto \bar{x} z \bar{y} \overline{1},
$$
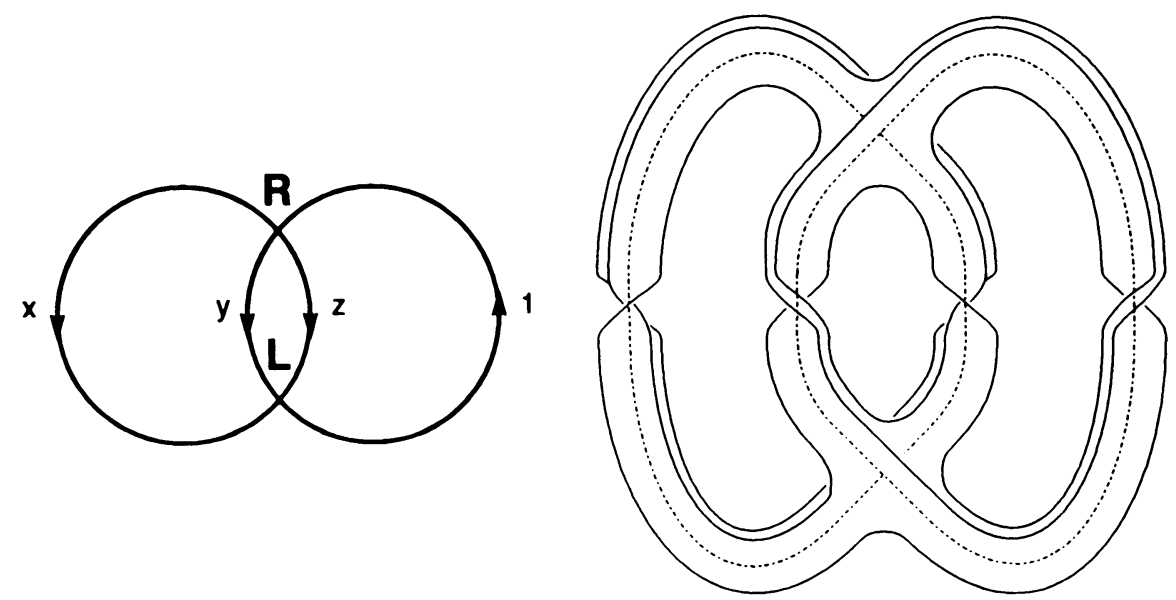

FIGURE 6.1. The swaddled graph and $\mathscr{Y}$ of the suspension of the DA of the Thom map 


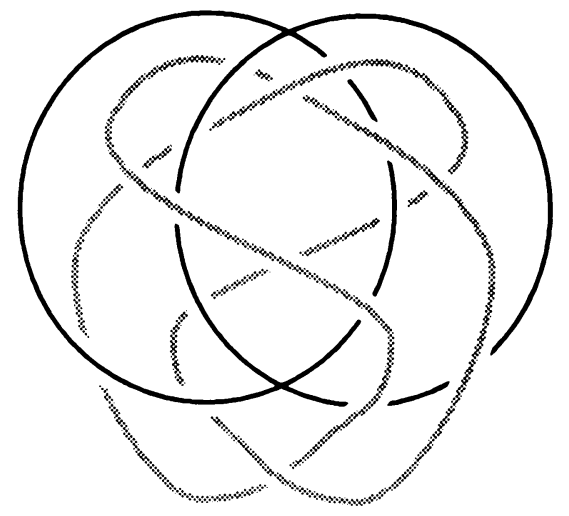

FIGURE 6.2. The suspension of the DA of the Thom map embeds in the complement of the figure eight knot in $\mathbf{S}^{3}$
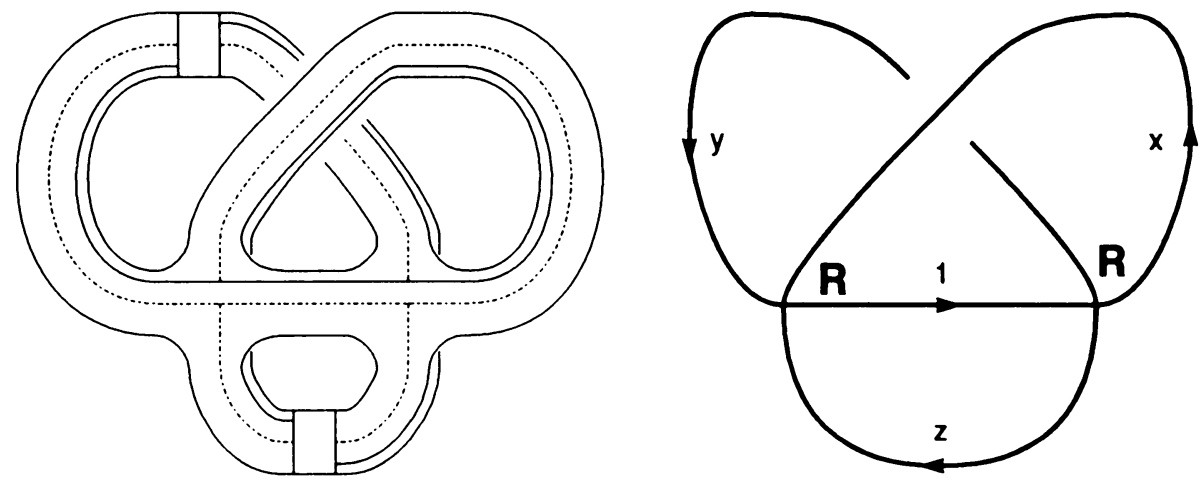

FIGURE 6.3. An attractor which is neither orientable nor transversely orientable. The boxes in the diagram of $\mathscr{Y}$ represent some odd number of half twists

where the overbar indicates that the orientation is reversed. Giving this the even punctuation

$$
\begin{aligned}
& \rho: x \mapsto y 1, \quad \rho: 1 \mapsto x \bar{z}, \\
& \rho: \bar{y} \mapsto \bar{x} z, \quad \rho: z \mapsto \bar{y} \overline{1},
\end{aligned}
$$

we see that $\phi$ is topologically transitive on $\Lambda$.

Our second swaddled graph, in Figure 6.3, has precisely one boundary periodic orbit, and comes from an attractor which is neither orientable nor transversely orientable. The underlying three-manifold is orientable and has fundamental group with the presentation

$$
\pi_{1}=\left\langle x, y, z \mid x \bar{z} x \bar{y}=1=x y \bar{z}^{2} y\right\rangle
$$

which reduces to

$$
\pi_{1}=\left\langle x, z \mid x^{2} \bar{z} x \bar{z}^{2} x \bar{z} x\right\rangle .
$$

While this manifold is an homology circle, it is not a knot complement in $\mathbf{S}^{3}$, since the homomorphism $x \mapsto 1, z^{2} \mapsto 1$ kills $\pi_{1}(\partial \mathbf{M})$, but does not kill 
$\pi_{1}(\mathbf{M})$. We owe this observation to Jon Simon. The return map is given as

$$
\rho: x \mapsto z \bar{x} y, \quad \rho: y \bar{z} \overline{1} \mapsto \overline{1} \bar{x} \overline{1} \bar{y} z .
$$

The third example, in Figure 6.4, comes from an orientable three-manifold with $H_{1}(\mathbf{M} ; \mathbb{Z})=\mathbb{Z} \oplus \mathbb{Z} / 5$. The fundamental group has the presentation

$$
\pi_{1}=\left\langle x, y, z \mid x \bar{z} y^{2} \bar{z}=1=x \bar{y} x z\right\rangle
$$

which reduces to

$$
\pi_{1}=\left\langle x, y \mid x^{2} \bar{y} x y^{2} x \bar{y} x\right\rangle .
$$

The first return map is

$$
\rho: x 1 \mapsto y \bar{x} \overline{1} \bar{z}, \quad \rho: y z \mapsto 1 y \bar{z} x .
$$

There is just one boundary periodic orbit.

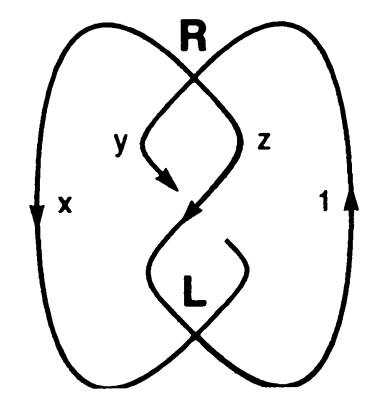

FIGURE 6.4. The isolating neighborhood of this attractor has torsion in its first homology
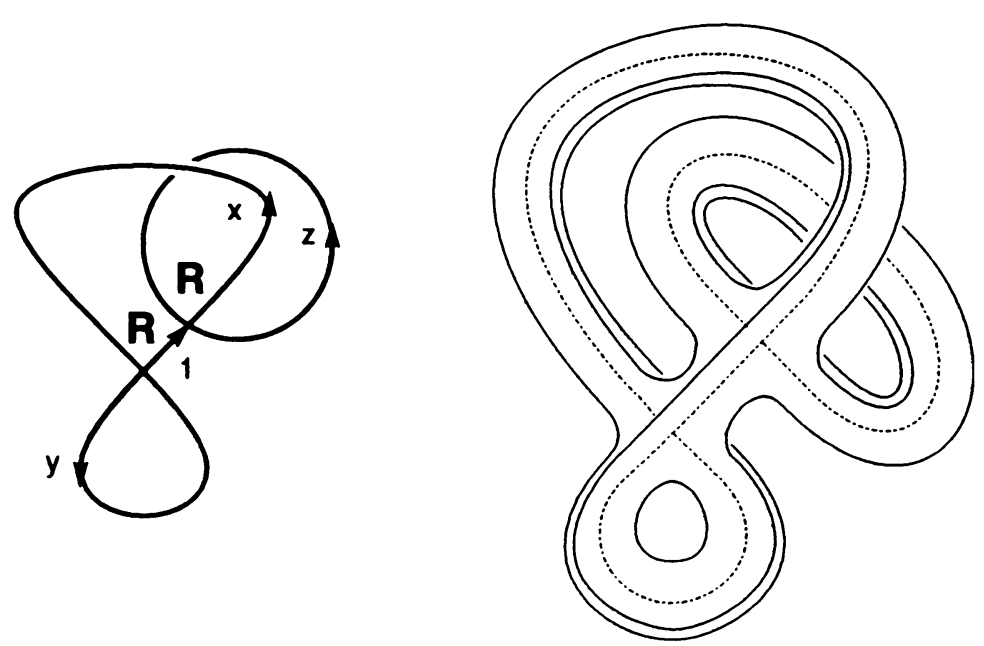

FIGURE 6.5. A swaddled graph with two dangling circles, one outgoing, at the bottom, and one incoming, on the upper right 
Our fourth example, in Figure 6.5, has a pair of dangling circles, and hence an annular sector when tidied up. When we have annular sectors there are four possible ways in which the corresponding cycles in $\mathbf{B}$ may be identified, according as the orientations of $\mathbf{W}$ along the cycles and the transverse orientations to $\mathbf{W}$ over the cycles are preserved or reversed. Only the orientations of $\mathbf{W}$ along the cycles affect the fundamental group of the exfoliated $\mathbf{M}$. The transverse orientations are reflected in the peripheral structure of $\mathbf{M}$, that is the injections of $\pi_{1}$ (component of $\partial \mathbf{M}$ ) in $\pi_{1}(\mathbf{M})$. The element $t$ in $\pi_{1}(\mathbf{M})$ which conjugates the dangling circles is only orientation preserving when exactly one of the two orientations above is reversed. Thus, with these four tidy swaddlings, we have four distinct attractors.

The $(-,-)$ tidying exfoliates to give the 2 component link complement in $\mathbf{S}^{3}$ shown in Figure 6.6, while the others can not embed in $\mathbf{S}^{3}$, because either they or their boundaries are nonorientable. The flow on the complement of the fibered link is not transverse to any fibration, since our link is not prime and hence has reducible monodromy, while our flow, being hyperbolic, would give pseudo-Anosov monodromy [Fr].

With the $\mathbf{W}$-orientation preserving glueing we get

$$
\pi_{1}=\langle x, y, z, t \mid x \bar{z} \bar{y}=1=x y \bar{x} \bar{z}, t y=z t\rangle
$$

which reduces to

$$
\pi_{1}=\langle z, t \mid \bar{t} z t z \bar{t} z t \bar{z} \bar{t} \bar{z} t \bar{z}\rangle \text {. }
$$

We leave it to the reader to check that if we set $t=1$ in the first presentation and eliminate $z$, then it reduces to the two bridge presentation of the trefoil, and that when we glue so as to preserve the transverse orientation as well, $z$ is a meridian, confirming our identification of the link. The first return map is

$$
\rho: 1 z \mapsto \bar{y} 1 x 1, \quad \rho: x \mapsto z x \bar{y}, \quad \rho: y \mapsto z .
$$
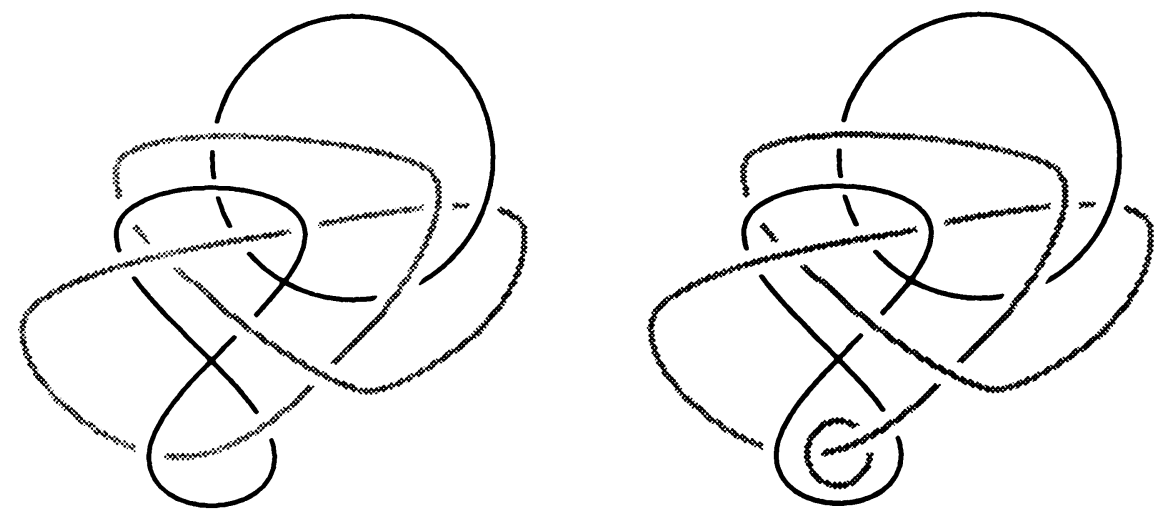

Figure 6.6. Before tidying, the swaddled graph embeds in the complement of a left trevoil. The dangling circles are both meridians and the $(-,-)$ tidying, the only one possible ambiently in $\mathbf{S}^{3}$, exfoliates to the link complement on the right. Note that the $\mathscr{Y}$ in Figure 6.5 must acquire a full right twist in the uppermost band for an embedding which extends across $\mathbf{W}$ 


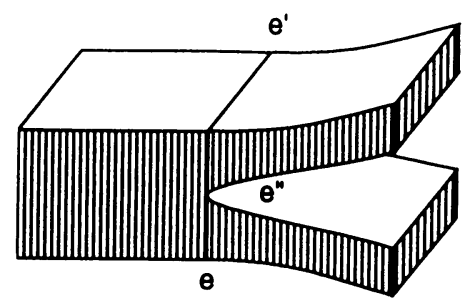

Figure 6.7. The three places (sector) $\times \partial \mathbf{I}$ glues together near an edge $e$ of $\mathbf{B}$ to form $\partial \mathbf{M}$
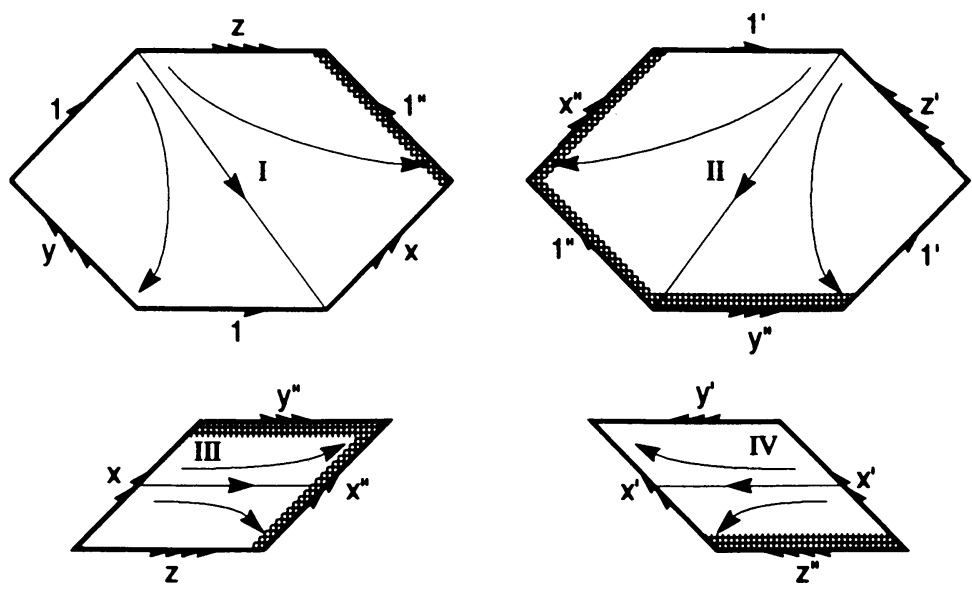

FIGURE 6.8. The regions $\partial \mathbf{I} \times$ sector in the boundary of the exfoliation of the swaddled graph in Figure 6.5. The maw is hatched.

With the $\mathbf{W}$-orientation reversing glueing we get

$$
\pi_{1}=\langle x, y, z, t \mid x \bar{z} \bar{y}=1=x y \bar{x} \bar{z}, t y=\bar{z} t\rangle
$$

which reduces to

$$
\pi_{1}=\langle z, t \mid \bar{t} \bar{z} t z \bar{t} \bar{z} t \bar{z} \bar{t} z t \bar{z}\rangle .
$$

The first return map is

$$
\rho: 1 z \mapsto \bar{y} 1 x 1, \quad \rho: x \mapsto z x \bar{y}, \quad \rho: y \mapsto \bar{z} .
$$

In either case, there are two boundary periodic orbits. To see the boundaries, we will carefully construct all four from the boundary of the three-manifold gotten by splitting $\mathbf{M}$ along the cylinder, fibered by $\mathscr{W}_{\text {loc }}^{s}$, over the core of the annular sector of $\mathbf{W}$. The boundary of this manifold is built up from two copies of each of the disc sectors, coming from the $\partial \mathbf{I}$ bundles over the sectors, and two annuli, coming from the cylinder along which we split $\mathbf{M}$. Now along each edge of $\mathbf{B}$, there are three ways in which the boundaries of the copies of the sectors may be glued in $\partial \mathbf{M}$, either above $\mathbf{B}$, below $\mathbf{B}$, or in the maw; above and below refer to some local transverse orientation to $\mathbf{W}$ along $\mathbf{B}$. See Figure 6.7. When we keep track of this data and perform all the glueings of the disc sectors we are left with two cylinders. The boundaries of the interval bundles over the sectors are illustrated in Figure 6.8; Figure 6.9 shows the boundary of M split open along the cylinder above. 


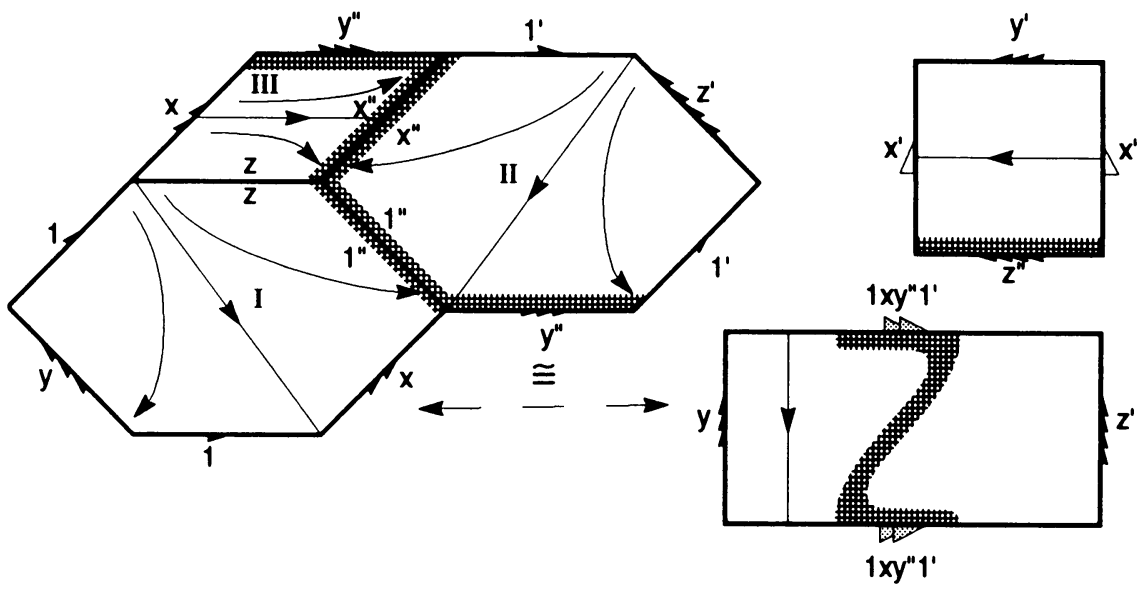

FIGURE 6.9. The boundary of the exfoliation of the swaddled graph in Figure 6.5
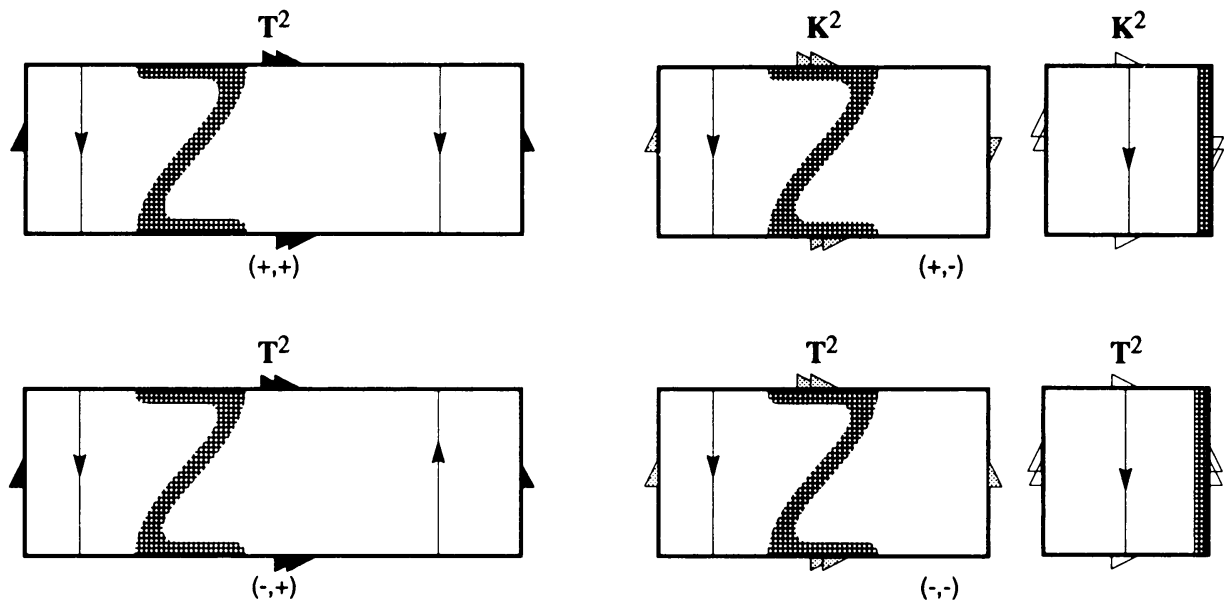

FIGURE 6.10. The boundaries of the four tidyings of the swaddled graph in Figure 6.5

The four possible ways to pair the four boundary circles come from the four tidyings of the dangling cycles of the swaddled graph $\mathbf{B}$, see Figure 6.10. In particular, when we reverse the orientation of $\mathbf{W}$ and preserve the transverse orientation, we obtain a nonorientable three-manifold with an incoherent attractor. To get an example on an orientable three-manifold, we need only take the double cover which has two copies of the trefoil knot complement glued each to the other as above. This answers the main open question from [C1].

The swaddled graph in Figure 6.11 is unrealizable in a dynamic branched surface, since it has quadruply tangent boundary circles.

From the swaddled graph in Figure 6.12, which differs from that in Figure 6.5 only in the parity of one crossing, come branched surfaces which carry ancompressible surface and one can check that in the dynamics there are a suspended 2-shift and either an attracting torus or Klein bottle, depending on how the dangling circles are paired. 


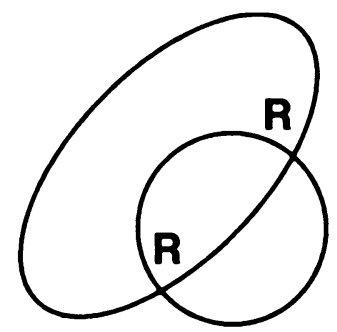

FIGURE 6.11. This branched surface has a single sector, so there must be four tangencies

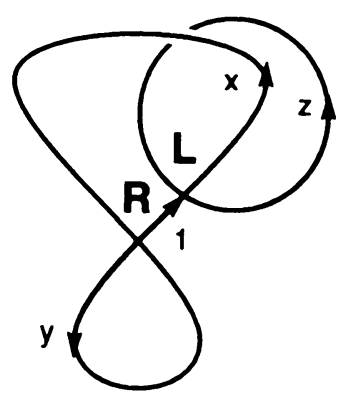

FIGURE 6.12. No matter how this swaddled graph is tidied up, it always carries an incompressible torus or Klein bottle
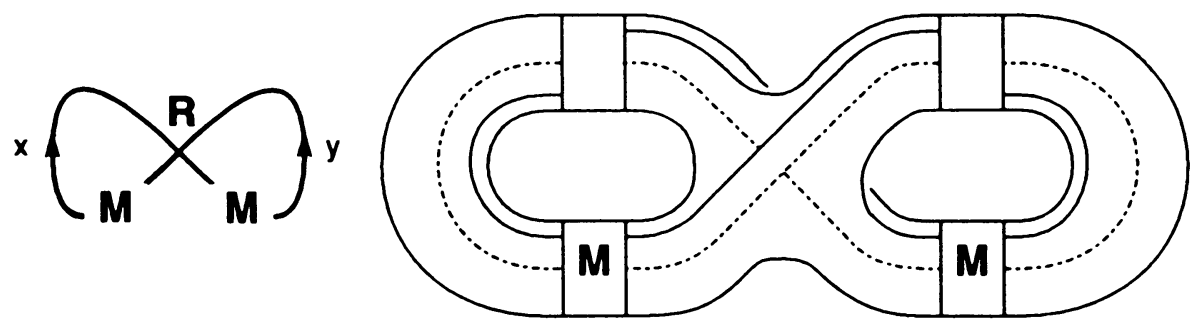

FIGURE 6.13. The isolating neighborhood of this attractor is nonorientable

When there are mirrors in the swaddled graph we can get attractors on nonorientable three-manifolds, as in our last example, in Figure 6.13.

Note that the orientation double cover is our first example on the figure eight knot complement. Here the fundamental group is $\left\langle x, y \mid x^{2} y \bar{x} \bar{y}^{2}\right\rangle$. The return map is given by $\rho: \bar{x} y \mapsto x y \bar{x} \bar{y}$. The boundary of $\mathbf{M}$ is a Klein bottle; the maw is a single orientation preserving curve which interleaves with the orientation reversing curves homotopic to the two boundary periodic orbits.

\section{APPENDIX}

Lemma. Up to the action of the mapping class group there are three isotopy classes of essential simple closed curves on $\mathbf{K}^{2}$. See Figure 4.3. 
Proof. Consider an essential simple closed curve $\mathscr{C}$; its normal bundle $\nu$ can be either a Möbius band or an annulus. In the first case, the complement $\mathbf{K}^{2} \backslash \mathscr{N}$ of a small tubular neighborhood is also a Möbius band. We can construct a homeomorphism of $\mathbf{K}^{2}$ to itself taking any simple closed curve $\mathscr{C}_{1}$ with twisted normal bundle to any other such curve $\mathscr{C}_{2}$ by first defining it on the complements of their tubular neighborhoods $\mathbf{K}^{2} \backslash \mathscr{N}_{1}$ and $\mathbf{K}^{2} \backslash \mathscr{N}_{2}$ and then taking an extension across the tubular neighborhood $\mathscr{N}_{1}$ which takes a collar of the boundary of $\mathbf{K}^{2} \backslash \mathscr{N}_{1}$ to $\mathscr{N}_{2}$ preserving the fiber structure on the the interior of the collar and inducing a double cover of $\mathscr{C}_{2}$ by the boundary of $\mathbf{K}^{2} \backslash \mathscr{N}_{1}$; this map is unique up to isotopy. In the second case, the complement of a tubular neighborhood is an annulus and there are now two ways to map the two collars of the boundary of $\mathbf{K}^{2} \backslash \mathscr{N}_{1}$ to $\mathscr{N}_{2}$ as above, according to the relative orientations.

\section{REFERENCES}

[AS] R. Abraham and S. Smale, The nongenericity of structural stability, Proc. Sympos. Pure Math., vol. 14, Amer. Math. Soc., Providence, R.I., 1970, pp. 5-8.

[C1] J. Christy, Intransitive Anosov flows on three-manifolds, Mem. Amer. Math. Soc. (to appear).

[C2] _ Branched surfaces and attractors. II: Naming names, (in preparation).

[C3] _ Immersing branched surfaces in dimension three, Proc. Amer. Math. Soc. (to appear).

[FO] W. Floyd and U. Oertel, Incompressible surfaces via branched surfaces, Topology 23 (1984), 117-125.

[Fr] D. Fried, Fibrations over $\mathbf{S}^{1}$ with pseudo-Anosov monodromy, Astérisque 66-67 (1979), 251-266.

[GW] J. Guckenheimer and R.F. Williams, Structural stability of Lorenz attractors, Inst. Hautes Études Sci. Publ. Math. 50 (1979), 59-72.

[He] J. Hempel, 3-manifolds, Princeton Univ. Press, Princeton, N.J., 1976.

[Hi] M. Hirsch, Differential topology, Springer-Verlag, New York, 1976.

[M] R. Mañé, A proof of the $C^{1}$ stability conjecture, Inst. Hautes Études Sci. Publ. Math. 66 (1989), 41-73.

[N] S. Novikov, Topology of foliations, Trans. Moscow Math. Society 14 (1965), 268-305.

[0] U. Oertel, Measured laminations in 3-manifolds, Trans. Amer. Math. Soc. 305 (1988), 531573.

[Pa] W. Parry, Symbolic dynamics and transformations of the unit interval, Trans. Amer. Math. Soc. 122 (1966), 368-378.

[P1] M. Peixoto, On structural stability, Ann. of Math. 69 (1959), 199-222.

[P2] - Structural stability on two-dimensional manifolds, Topology 1 (1962), 101-120.

[Rou] R. Roussarie, Plongements dans les variétés feuilletées et classification de feuilletages sans holonomie, Inst. Hautes Études Sci. Publ. Math. 43 (1974), 101-142.

[Rob] R. C. Robinson, Structural stability of vector fields, Ann. of Math. 99 (1974), 154-175.

[Sh] M. Shub, Global stability of dynamical systems, Springer Verlag, New York, 1986.

[Sm1] S. Smale, On dynamical systems, Bol. Soc. Mat. Mexicana (2) 5 (1960), 195-198.

[Sm2] _ The mathematics of time, Springer Verlag, New York, 1980.

[Ta] F. Tangerman, Topological conjugacy for correspondences, personal communication.

[T] W. Thurston, Earthquakes in two-dimensional hyperbolic geometry, Low Dimensional Topology and Kleinian Groups (D. B. A. Epstein, ed.), Cambridge Univ. Press, Cambridge, 1986, pp. $91-112$. 
[Wald] F. Waldhausen, On irreducible 3-manifolds which are sufficiently large, Ann. of Math. 87 (1968), 56-88.

[Wms] R. F. Williams, Expanding attractors, Inst. Hautes Études Sci. Publ. Math. 43 (1973), 473-487.

Department of Mathematics, University of California, Santa Cruz, California 95064 Current address: Mathematical Sciences Research Institute, 1000 Centennial Drive, Berkeley, California 94720

E-mail address: joe@msri.org 